\title{
Cutting a Convex Polyhedron Out of a Sphere
}

\author{
Syed Ishtiaque Ahmed, Masud Hasan, and Md. Ariful Islam \\ Department of Computer Science and Engineering \\ Bangladesh University of Engineering and Technology \\ Dhaka 1000, Bangladesh \\ ishtiaque@csebuet.org,masudhasan@cse.buet.ac.bd, arifulislam@csebuet.org
}

\begin{abstract}
Given a convex polyhedron $P$ of $n$ vertices inside a sphere $Q$, we give an $O\left(n^{3}\right)$-time algorithm that cuts $P$ out of $Q$ by using guillotine cuts and has cutting $\operatorname{cost} O\left((\log n)^{2}\right)$ times the optimal.
\end{abstract}

\section{Introduction}

The problem of cutting a convex polygon $P$ out of a piece of planar material $Q$ ( $P$ is already drawn on $Q$ ) with minimum total cutting length is a well studied problem in computational geometry. The problem was first introduced by Overmars and Welzl in 1985 [9] but has been extensively studied in the last eight years [1-7,9-11] with several variations, such as $P$ and $Q$ are convex or non-convex polygons, $Q$ is a circle, and the cuts are line cuts or ray cuts. The results include: indication to the hardness of optimality, several $O(\log n)$ and constant factor approximation algorithms and a PTAS. See [1] for a summary of these results.

The generalization of this problem in $3 \mathrm{D}$ is very little known. To the best of our knowledge, the only result is to decide whether a polyhedral object can be cut out form a larger block using continuous hot wire cuts [7].

This type of cutting problems have many industrial applications such as in metal sheet cutting, paper cutting, furniture manufacturing, ceramic industries, fabrication, ornaments, and leather industries. Some of their variations also fall under stock cutting problems [3].

In this paper we consider the problem of cutting a convex polyhedron $P$ which is fixed inside a sphere $Q$ by using only guillotine cuts with minimum total cutting cost. A guillotine cut, or simply a cut, is a plane that does not pass through $P$ and partitions $Q$ into two smaller convex pieces. After a cut is applied, $Q$ is updated to the piece that contains $P$. The cutting cost of a guillotine cut is the area of the newly created face of $Q$. We give an $O\left(n^{3}\right)$-time algorithm that cuts $P$ out of $Q$ by using only guillotine cuts and has cutting cost no more than $O\left((\log n)^{2}\right)$ times the optimal cutting cost.

\section{Algorithm}

The overall idea is as follows. Let $C^{*}$ be the optimal cutting cost. We shall have two phases in our algorithm: $b o x$ cutting phase and carving phase. In the box cutting phase, we shall cut a minimum volume rectangular box $B$ containing $P$ out of $Q$ with cutting cost no more than a constant factor of $C^{*}$. Then in the carving phase we shall cut $P$ out of $B$ with cutting cost bounded by $O\left((\log n)^{2}\right)$ times of $C^{*}$.

A cut is vertex/edge/face cut if it is tangent to $P$ at a vertex/edge/face respectively. We call $P$ to be cornered if it does not contain the center $o$ of $Q$, otherwise it is called centered. For cornered $P$, the $D$-separation of $P$ is the minimum-cost (single) cut that separates $P$ from $o$. A point $p$ of $P$ is visible from $o$ if the line segment $\overline{o p}$ does not intersect any other point of $P$.

\subsection{Box cutting phase}

If $P$ is cornered, we first apply a D-separation to $Q$.

Lemma 1. The D-separation must be either a vertex, edge or face cut. Moreover, if $\overline{\mathrm{oO}^{\prime}}$ is the line segment perpendicular to the D-separation at $o^{\prime}$, then $o^{\prime}$ must be the corresponding vertex or a point of the corresponding edge or face.

Proof. Let $x$ be the closest point of $P$ from $o$. Clearly, $x$ is visible from $o$. A D-separation must be the plane that can separate $o$ from $x$ and is furthest from $o$. This plane is none but the plane perpendicular to $\overline{o x}$ at $x$. This plane is also tangent to $P$, since otherwise $x$ would not be closest to $o$.

Lemma 2. The D-separation can be found in $O(n)$ time. 
For cornered $P$, after the D-separation is applied, let $r$ be the radius of the base circle of $Q$.

Lemma 3. For cornered $P$, cost of the D-separation, which is $\pi r^{2}$, is at most $C^{*}$.

Proof. [Sketch only] The proof depends upon the fact that the cuts in an optimal cutting sequence must be tangents to $P$. Overmars and Welzl [9] proved this fact for 2D, whose 3D generalization also holds. The idea is that if $c$ is the first cut that does not touch $P$, then the cost of $c$ and the subsequent cuts behaves, while moving $c$ parallelly, as a concave function in the distance of $c$ from $P$. Therefore, the minimum cost is achieved when it touches $P$ or is infinitely away from $P$. With the above fact, the authors in [1] proved in 2D that to separate $P$ from $o$ an optimal cutting sequnce must use the D-separation or use cuts with cost higher than the D-separation. The 3D generalization of this proof also holds.

Lemma 4. For centered $P, C^{*} \geq \pi R^{2}$, where $R$ is the radius of $Q$.

Proof. [Sketch only] Orthogonally project $P$ and $Q$, where $Q$ projects to a circle $C$ and $P$ to a convex polygon $X$ inside $C$. Any optimal cutting sequence must have cost at least the area of $X$ plus the difference of the area of $C$ and $X$.

We next find a minimum volume rectangular bounding box $B$ of $P$ in $O\left(n^{3}\right)$ time by the algorithm of O'Rourke [8]. Then we cut out this box from $Q$ by applying six cuts along the six faces of $B$.

Lemma 5. Cost of cutting $B$ out of $Q$ is at most $3 C^{*}$ for cornered $P$ and at most $4 C^{*}$ for centered $P$.

Proof. [Sketch only] Let $S$ be the surface of $Q$. For cornered $P$, area $|S|=3 \pi r^{2} \leq 3 C^{*}$ (by Lemma 3) and for centered $P,|S|=4 \pi R^{2} \leq 4 C^{*}$ (by Lemma 4). While cutting along the faces of $B$, for each cut $c$ let $Q^{\prime}$ be the portion of $Q$ that does not contain $P$. Let $q^{\prime}$ be the portion of the surface of $Q^{\prime}$ that is "inherited" from $S$. One important observation is that the cost of $c$ is no more than the area of $q^{\prime}$. Moreover, over all six cuts, sum of these inherited surface area is at most $S$. Therefore, the lemma holds.

Lemma 6. $C^{*} \geq \frac{1}{3}|B|$, where $|B|$ is the area of $B$.

Proof. [Sketch only] Let $g$ be a maximum area face of $B$. Project $P$ orthogonally from the direction perpendicular to $g$. $P$ projects to a convex polygon $X$. Observe that in this projection, $g$ is the minimum area bounding rectangle of $X$, since otherwise we could rotate the four faces of $B$ that are not perpendicular to $g$ and would get a bounding rectangle smaller than $g$, which in turn would give a bounding box smaller than $B$. It implies that the area of $X$ is at least $\frac{1}{2}|g|$. Now, $C^{*}$ is at least twice the area of $X$, and $|B| \leq 3|g|$. Therefore, $C^{*} \geq \frac{1}{3}|B|$.

\subsection{Carving phase}

Let $T=B-P$ be the portion of $B$ that is "trapped" between the boundaries of $P$ and $B . T$ is a polyhedral object, convex or non-convex and possibly disconnected. The inner (outer) surface of $T$ is the surface that touches (does not touch) the faces of $P$. Our idea is to apply an edge cut through each edge of $P$, and we shall do that in two types of rounds: face rounds and edge rounds. Face rounds will find polygonal chains that will partition the faces of $P$ into smaller connected components and edge rounds will apply edge cuts through the edges of those polygonal chains. There will be $O(\log n)$ face rounds. Within each face round there will be a number of edge rounds but their total cost will be $O\left(C^{*} \log n\right)$. Once we have applied edge cuts through all the edges of $P$, each face $f$ of $P$ will have a small "cap"-like portion of $T$ over it, which we shall cut at a cost of the area of $f$ to get $P$, giving a cost of $O\left(C^{*}\right)$ for all faces.

Face rounds Let $F$ be a connected face set of $m$ faces of $P$. At the very first face round $i=0, F$ consists of all the faces of $P$. We find a chain of edges $P^{\prime}$ that will partition $F$ into two smaller connected face sets $F_{1}$ and $F_{2}$ by the following lemma. (Precise definition of "connected" needs detail discussion, which we omit in this extended abstract.)

Lemma 7. It is always possible to find an orthogonal projection of $P$ which is non-degenerate w.r.t the faces of $F$ such that the sets of visible and invisible faces of $F$ contain at least $\left\lfloor\frac{m}{2}\right\rfloor$ faces each. 
Proof. [Sketch only] For this proof we shall move on to the surface of an origin-centered sphere $s$. For each face $f \in F$, its outward normal is uniquely represented by a point of $s$, which we call the normal point of $f$. Each point of $s$ also represents an orthogonal projection direction of $P$. So, an orthogonal projection of $P$ which is non-degenerate w.r.t the faces of $F$ is represented by a great circle of $s$ that does not pass through the normal points of the faces of $F$. We need one such great circle satisfying an additional criterion that its two hemispheres contain at least $\left\lfloor\frac{m}{2}\right\rfloor$ normal points each. There exists infinitely many such great circles and one of them can be found in $O(n \log n)$ time.

$P^{\prime}$ is the chain of edges in the boundary of the above projection whose each edge has both adjacent faces (one is visible and another is invisible) in $F$. We call $P^{\prime}$ a separating chain of $F$. We shall apply edge cuts through the edges of $P^{\prime}$ by the edge rounds as described in the next paragraph. In the next face round $i=1$, we shall apply Lemma 7 for each of $F_{1}$ and $F_{2}$ and shall thus get two separating chains and four connected face sets. We shall repeat the same procedure for each of these four face sets. We shall continue like this until each face set has only one face. Clearly, we need $O(\log n)$ face rounds.

Edge rounds Let $P^{\prime}=e_{1}, e_{2}, \ldots, e_{k}$ with its two ends from $e_{1}$ abd $e_{k}$ touching the outer surface of $T$. We shall apply edge cuts through the edges of $P^{\prime}$ such that all of them are parallel to a particular direction. Such a direction can be the corresponding projection direction. We call this set of $k$ edge cuts a zone of cuts and its direction the zone cut direction. We shall apply these cuts in $\log k$ edge rounds. At the very first edge round $j=0$, we apply an edge cut through $e_{k / 2}$ in the zone cut direction. This cut will partition the edges of $P^{\prime}$ into two subchains of size at most $\left\lfloor\frac{k}{2}\right\rfloor$. In the next round, we apply two edge cuts through the two middle edges of these two subchains, which will result into four subchains. Then in the next round we apply four similar cuts to the four subchains. We continue like this until each subchain has only one edge. Clearly, we need $O(\log k)$ edge rounds for $P^{\prime}$.

Lemma 8. After all the face rounds and the corresponding edge rounds are completed, all edges of $P$ gets an edge cut.

Proof. Let $e$ be an edge that does not get an edge cut. Then the two adjacent faces of $e$ are in the same face set. But that is a contradiction that each face set has only one face.

Analysis We define the box area of a face set $F$ as follows. When $F$ contains all faces of $P$, its box area is $B$ - the whole surface area of $B$. Zone of cuts through the separating chain of $F$ partitions $F$ into $F_{1}$ and $F_{2}$ and $T$ into two components, say $T_{1}$ and $T_{2}$, respectively. Then the box area of $F_{1}\left(F_{2}\right)$ is the outer surface area of $T_{1}$ $\left(T_{2}\right)$, which we denote by by $B_{1}\left(B_{2}\right)$. Observe that $\left|B_{1}\right|+\left|B_{2}\right| \leq|B|$. Box area of any subsequent face set is similarly defined. Moreover, two face sets from the same face round have their box areas disjoint and in any face round sum of all box area is at most $|B|$.

Lemma 9. Let $P^{\prime}{ }_{m}$ be the separating chian with $k$ edges of an arbitrary face set to which we apply $O(\log n)$ edge rounds. Let $B_{m}$ be the box area of $F_{m}$. At each edge round $j$, total cost of $2^{j}$ cuts is $O\left(\left|B_{m}\right|\right)$. Over all $\log k$ edge rounds, total cost is $\left(\left|B_{m}\right| \log n\right)$.

Proof. [Sketch only] This proof is similar to that of Lemma 5, Consider a particular edge round $j$. For each cut $c$ the cost of $c$ is no more than the portion of $\left|B_{m}\right|$ that is thrown away by $c$. Moreover, these cuts are pairwise disjoint. Indeed, they can at best intersect the cut which is in between them and was applied in $(j-1)$-th round. It implies that the total cost of $2^{j}$ cuts is at most $\left|B_{m}\right|$. Since $k \leq n$, the second part of the lemma follows.

Lemma 10. Let $F$ be the face set consisting of all faces of $P$ to which we shall apply $O(\log n)$ face rounds. At each face round $i$, total cost of $2^{i}$ zones of cuts is $O(|B| \log n)$. Over all $O(\log n)$ face rounds, the total cost is $O\left(C^{*} \log n\right)$.

Proof. At each face round $i$, we apply $2^{i}$ zones of cuts to $2^{i}$ face sets. By the previous lemma, for a particular face set $F_{m}, 0 \leq m \leq 2^{i}$, cost of the zone of cuts applied to it is at most $O\left(\left|B_{m}\right| \log n\right)$. Since $\sum_{1}^{2^{i}}\left|B_{m}\right| \leq|B|$, cost of all zone cuts is $\sum_{1}^{2^{i}} O\left(\left|B_{m}\right| \log n\right)=O(|B| \log n)$. Over all $O(\log n)$ face rounds, the total cost is $O\left(|B|(\log n)^{2}\right)$, which by Lemma6 is $O\left(C^{*}(\log n)^{2}\right)$.

Theorem 1. Given a convex polyhedron $P$ fixed inside a sphere $Q, P$ can be cut out of $Q$ by using only guillotine cuts in $O\left(n^{3}\right)$ time with cutting cost $O\left((\log n)^{2}\right)$ times the optimal, where $n$ is the number of vertices of $P$. 


\section{References}

[1] S. I. Ahmed, M. A. Islam, and M. Hasan. Cutting a cornered convex polygon out of a circle. J. Computers, to appear.

[2] S. Bereg, O. Daescu, and M. Jiang. A PTAS for cutting out polygons with lines. In COCOON'06, LNCS-4112:176-185, 2006.

[3] J. Bhadury and R. Chandrasekaran. Stock cutting to minimize cutting length. Euro. J. Oper. Res., 88:69-87, 1996.

[4] R. Chandrasekaran, O. Daescu, and J. Luo. Cutting out polygons. In CCCG'05, pp. 183-186, 2005.

[5] E. D. Demaine, M. L. Demaine, and C. S. Kaplan. Polygons cuttable by a circular saw. CGTA, 20:69-84, 2001.

[6] A. Dumitrescu. An approximation algorithm for cutting out convex polygons. CGTA, 29:223-231, 2004.

[7] J. W. Jaromczyk and M. Kowaluk. Sets of lines and cutting out polyhedral objects. CGTA, 25:67-95, 2003.

[8] J. O'Rourke. Finding minimal enclosing boxes. Int. J. Comp. Inform. Sci., 14(3):183-199, 1985.

[9] M. H. Overmars and E. Welzl. The complexity of cutting paper. In SoCG'85, pp. 316-321, 1985.

[10] X. Tan. Approximation algorithms for cutting out polygons with lines and rays. In COCOON'05, LNCS-3595:534-543, 2005.

[11] G. Toussaint. Solving geometric problems with the rotating calipers. In MELECON'83. Athens, Greece, 1983. 